\title{
Evaluation on Equity and Efficiency of Financing for Total Health Expenditure in China
}

\author{
Weiwei Liu ${ }^{1, \mathrm{a}}$ and Han $\mathrm{He}^{2, \mathrm{~b}, *}$ \\ ${ }^{1}$ School of Public Health and Management, Chongqing Medical University, Chongqing 400016, \\ China \\ ${ }^{2}$ Rongzhi College of Chongqing Technology And Business University, Chongqing 400000, China \\ aweiweiliu521@hotmail.com, bgene771771@hotmail.com \\ *The corresponding author
}

Keywords: Total Health Expenditures in China; Health Financing; Efficiency and Equity; Evaluation Method

\begin{abstract}
To systematically summarize and analyze the efficiency and equity evaluation of health financing, and focus on the evaluation methods and contents of Chines health expenditure evaluation index. The literature were collected and summarized. The health expenditure evaluation index on inductive analysis of perspective can be divided into three parts, from the required data, calculation method, the applicable scope, the dimensions measurement of health financing equity and efficiency are analyzed. A rigorous and complete system of equity and efficiency of health financing in China is developed.
\end{abstract}

\section{Introduction}

WHO defines the health system as all organizations, institutions and resources devoted to improving the health of the population in the 2000 World Health Report: improving performance, and proposes a complete framework for health performance assessment, in which health financing is the core module of the health system. The basis for measuring the health performance of each member country. The health system has three major goals ${ }^{[1]}$, and the equity of health financing is the most important part. In the 2000 World Health report, WHO statistics the ranking of health financing equity in all countries, with total of 192 members and China was in the last fourth. To an extent, there is a big gap between China and the international equity financing system for health care, which has prompted China's health policy to constantly adjust and deepen medical reform. In the health financing system, the ultimate goal is improving the level of health and fairness. To optimize the structure of health financing, reduce the cost of additional expenses in the operation of various departments, raise the efficiency of financing, and improve the equity of health financing in some degree.

\section{Equity and Efficiency Evaluation Framework of China's Total Health Expenditure}

Theoretical Framework. Health expenditure is an important part of evaluating the equity and efficiency of health care system. This study summarizes the related health cost evaluation literature, classifies the evaluation index of health total cost, mainly into three aspects: financing level, financing equity and efficiency, and the relevant methods are discussed and analyzed in each aspect.

Financing Level. The financing level is an aspect of the evaluation of health expenditure, which focuses on the degree of concern about the health in the country or region, and holds an important position in coordinating health and economic development.

Financing level is the basic part of the health expenditure evaluation system, to a certain extent, it reflects the fairness of financing[6]. This paper mainly evaluates the investment of the whole society on health, combined with the factors affecting the results of the level of population and economic development, compare and analyze the results of the total health expenditure in each 
country, evaluate the social input to health and the coordination degree of health input in the economic development, the level of per capita health expenditure comparability, etc..

Financing Equity. The equity of health financing should include two key factors: 1) the risk sharing between the healthy person and the patient; 2) the risk sharing among the people of different income and different economic levels. In principle, 'rich people pay more' and 'poor people pay less' [8] . That is, on the health expenditure range, the rich bear more medical expenses [7].

On the basis of government, society and individual parties, the research on the equity of financing, combined with the policy strategy, the calendar year data and the regional development and changes, calculated and analyzed the health financing equity evaluation index.

Financing Efficiency. The efficiency of health financing is to search for the flow of total health expenses in the health financing system, such as the flow of funds in different types of institutions, such as hospitals, primary medical institutions and public health institutions. And the proportion of more funds raising in public health institutions and primary medical institutions reflects the health equity to a certain extent. For provincial accounting, the total health expenditure in different districts and counties can reflect the government's emphasis on health in different regions.

\section{Evaluation Index and Method}

The concentrated curve and the Lorenz curve method. The Kakwani index has some limitations to evaluate the progressive degree of health financing, and the mapping can only reflect and objectively reflect the distribution characteristics of different populations.

When the Lorenz curve is further applied, the concentration curve can be obtained when the financing is calculated. When expressing the concentration curve, the abscissa is the cumulative percentage of population and the ordinate is the cumulative percentage of financing, as follows Figure 1.

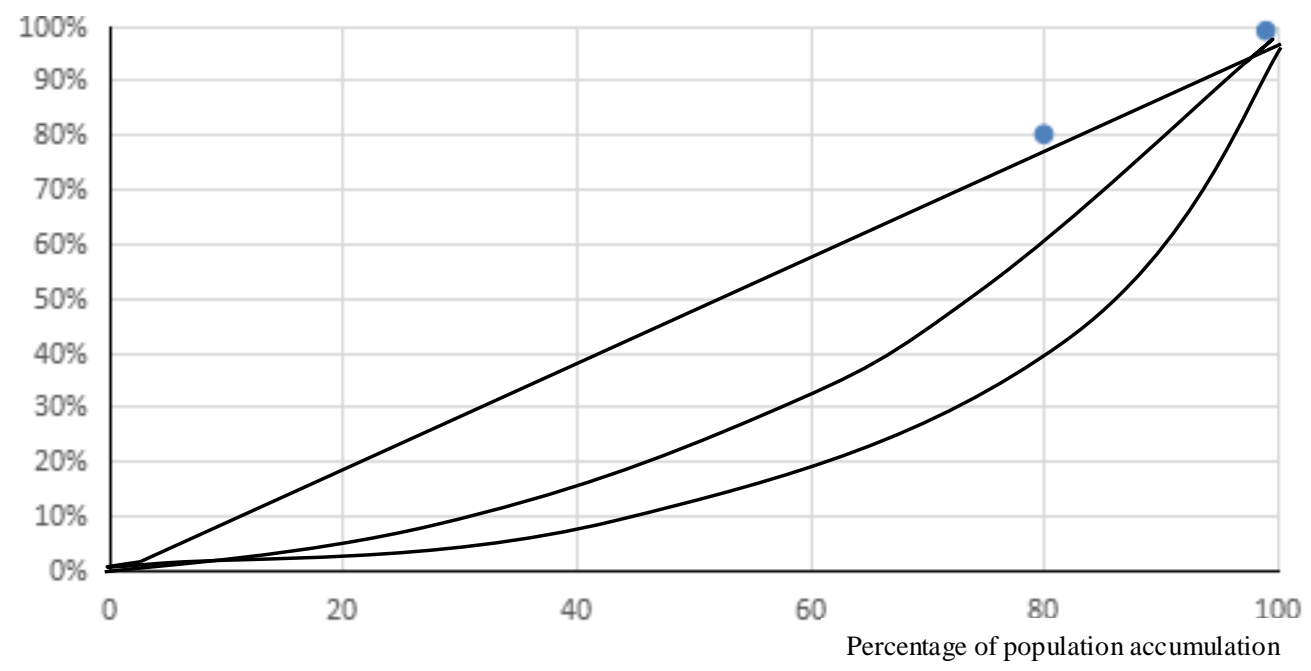

Figure 1 Lorenz curve and concentrated curve

From the figure 1 analysis, if the concentration curve and the Lorenz curve cross in the different population, it reflects the complexity of the crowd. If the Lorenz curve and the concentrated curve overlap, the financing channel is stable, neither progressive nor retrogressive. If the concentration curve is under the Lorenz curve, the channel is progressive and vice versa.

Benefit from Government Expenditure. From the perspective of fairness, government health expenditure should be tilted to low-income groups, playing a role in redistribution and regulating social equity. The benefit fairness analysis of government health expenditure is an international method to measure the fairness of the benefit of government health expenditure. This method combines the cost of public service or the information of unit subsidy and service utilization to measure and analyze the situation of the residents' benefit distribution to the actual population of government health expenditure. 
The index method uses the concentration index and Kakwani index to measure the benefit level of the residents. The concentration index reflects the absolute fairness and the concentration index is positive. It shows that the high income residents have obtained more government subsidies, and the concentration index is negative. The Kakwani index reflects relative fairness, and the Kakwani index is positive, reflecting the disadvantage of the distribution of government subsidies. In the poor, the Kakwani index is less than zero, reflecting that the government has subsidized the medical and health services for the poor.

As a public financial act, government health expenditure is also a means of social redistribution. It not only plays an important role in the development of medical institutions and the improvement of residents' health, but also improves the level of health, reduces the difference between regions and increases the social public degree.

Impartiality of Residents' Economic Impact. The fairness of the residents' economic impact is studied from two aspects:

Poverty impact: the standard of poverty impact research is that in a fair health financing system, undeserved residents are impoverished because of medical and medical costs for medical treatment, and the study of the impact of poverty is based on the family economic level and OOP (out-of-pocket) as the key variable, combined with local poverty standards, to calculate people's medical payment. The change of poverty gap index before and after health expenditure reflects the breadth and intensity of poverty caused by illness.

Catastrophic health expenditure: in a fair health financing system, there should be no residents to pay a serious impact on the household consumption structure because of the payment of medical and health expenses. That is, the proportion of OOP to household consumption should not exceed a specific standard (such as the $40 \%$ standard used by the WHO), which is OOP and family cancellation. The cost of expenditure (or payment capacity) is a variable by calculating the ratio of OOP in the population that accounts for more than the specific standard of household expenditure, the degree of health expenditure, the incidence of catastrophic health expenditure, and the probability and intensity of the catastrophic health expenditure of the population.

Total Health Expenditure in Urban and Rural areas, per capita Total Health Expenditure in Urban and Rural areas, and Medical Burden for Urban and Rural Residents. According to various factors, such as the economic level, cultural customs and health financing structure among different regions, a set of closely integrated health cost evaluation index system is formed to adapt to the local data collection, data analysis and policy release. Although the accounting of urban and rural health financing is subordinate to the total health cost accounting system, Due to the uneven level of the research level of health cost accounting in various regions, it is necessary to accurately calculate the total health costs in various regions and to establish a health cost evaluation system, which can effectively improve the technical level of the local health researchers.

\section{Financing Efficiency Evaluation Method}

The Flow of Total Health Expenditure to the Institutions. The total health expenditure flows is to hospitals, primary health care institutions, public health institutions, drug retail establishments, health administrative agencies and other health costs. In order to improve the equity of health, it is necessary to let health funds flow to public health institutions and primary medical institutions.

The proportion of public health institutions and primary health care institutions account for the total health expenditure, to a certain extent, reflects the benefit of the population in the public health care, and helps to improve health equity.

Total Health Expenditures flows in the Province. The flow of total health expenditure in provincial institutions is: for provincial accounting, total health expenditure flow in the sub provincial region, for municipal accounting, it refers to the flow of total health expenditure between different districts and counties in the city. The total health expenditure was calculated from two angles of source law and institutional law.

The evaluation index of financing efficiency is: the flow of total health expenditure and the flow of health expenditure in the province. The flow of total health expenditure includes hospital 
expenses, outpatient service expenses, drug retail agencies, public health institutions, health administration and medical insurance management, and other health expenses. More flow to public health institutions, the health expenditure of primary health care institutions will help to improve fairness, the proportion of health funds in public health institutions and primary health care institutions, and to extent, benefit in public health.

Taking the percentage of health expenditure in various institutions as an example from 2012 to 2014, as following Table 1.

Table 1 The percentage of Health Expenditure in Institutions from 2012 to 2014

\begin{tabular}{ccccc}
\hline & Year & 2012 & 2013 & 2014 \\
\hline \multirow{4}{*}{$\begin{array}{c}\text { Hospital } \\
\text { expenses }\end{array}$} & Total & 62.15 & 62.56 & 62.53 \\
\cline { 2 - 4 } & Public Hospital & 39.09 & 39.43 & 39.95 \\
\cline { 2 - 4 } & County Hospital & 14.09 & 14.31 & 14.29 \\
\cline { 2 - 4 } & Community Health Centre & 2.75 & 2.38 \\
\cline { 2 - 4 } & Township Hospital & 6.11 & 6.03 & 5.81 \\
\cline { 2 - 4 } & Other Hospitals & 0.11 & 0.10 & 0.09 \\
\hline Outpatient Cost & 8.00 & 8.00 & 7.68 \\
\hline \multicolumn{2}{r}{ Drug Retail Agency Costs } & 12.28 & 12.28 & 11.91 \\
\hline Health Administration and Medical Insurance Agency Costs & 7.49 & 7.49 & 7.41 \\
\hline
\end{tabular}

In 2012, the proportion of public health agency costs accounted for $7.49 \%$ of the total health cost, which was the highest proportion of the public health institutions from 2012 to 2014. It showed that the government paid more attention to public health in this year, and the population benefited more in public health. To some extent, health equity was reflected to some extent.

\section{Problems and Suggestions}

The Single Index of Evaluation is Biased. In the evaluation of health expenditure, the assessment of the total cost of health expenditure in the financing efficiency index can reflect the health equity to a certain extent, indicating the correlation between some indicators. If only a single indicator is used to evaluate the health expenditure, there may sometimes be an incomplete interpretation of the results, and the proposed policy recommendations may also have a certain deviation. Therefore, several indicators can be combined to discuss from the government, society and individuals, and put forward more reasonable and accurate suggestions.

The combination of the indicators can be make a comparison and analysis of the chart, but the evaluation index of health expenditure should always be combined with the policy needs and economic development and other background factors. It can not be divorced from the actual situation, only from the data, so as to get a fair evaluation for the national conditions and social benefits.

Evaluation is Influenced by Various Factors such as Economy and Politics. The fairness of health financing has long been paid attention to all over the world. With the development of China's economy and the continuous innovation and improvement of the market system, the accounting and evaluation of health expenditure in China need to be more fair and impartial, and the efficiency of the use of health funds has always been a key research subject in China, based on the health policy and medical treatment issued by our country. The deepening of the reform system will enhance the fairness of health. But in solving the differences in urban and rural health services, the poor look down on the disease and other issues, still need more government investment, and guided policies. By analyzing the causes of unfair and introducing corresponding health policies, we can solve the problem of inequity more reasonably.

The Collection of Evaluation Data is Difficult and the Accuracy is not High. The research on equity and efficiency of health financing needs a lot of accurate and available data, and how to 
improve the accuracy of data is the most important issue. In general, the research on the fairness of health financing should be based on the progressive research in various places. The study mainly focuses on the calculation of the Kakwani index and the drawing of the Lorenz curve, which quantifies the vertical equity of health financing in a region, and the key lies in the authenticity of the data collection. Many evaluation indicators need a large number of accurate data analysis, but China has not set up perfect and unified data collection system for health financing fairness evaluation, so the data collection is generally far from low accuracy and difficult. Comparatively speaking, the research on the fairness of health financing in China has established its own theory and research methods according to international theories and methods. However, it is difficult to combine the health evaluation innovation with the national conditions, and it is necessary to promote and try more research on health expenditure evaluation.

\section{Acknowledgements}

Supported by Science and Technology Research Project of Chongqing Education Commission (KJ1600240) and Humanities and Social Sciences Research Project of Chongqing Education Commission (17SKG018) .

\section{References}

[1] Z.Y.Guo, W.X.Shi, Study on equity of urban health financing in Guangxi Zhuang Autonomous Region, Modern Preventive Medicine.2013,28(1):269-271

[2] Y.M, X.M.Li, Analysis on the fairness evaluation method of health financing, Soft Science of Health. 2014,28(9):274-280

[3] S.H.Yang, Study on the accounting of total health expenditure (source of financing) in Guangxi from 2007 to 2009, Guangxi Medical University,2012

[4] Y.Y, Y.C,Q.M, Analyzing the progressivity of health financing in Dali Cit, Chinese Health Service Management, 2015,15(6):446-448

[5] Q.W, Y.X.Zhao, H.F, Research on the vertical fairness and progressien of health financing, Chinese Health Economics 2003,22(3):4-6

[6] J.W, J.J.Sun, F.H.Yu, Discussing the basic theoretical framework of sub-national total health cost accounting research sampled with Shangdong Province, Chinese Health Service Management, 2010(15):4-5

[7] S.X.Yang, Analysis and countermeasure research on the current situation of total health expenses financing, Medical University Of Tianjin, 2013

[8] X.W.Man, Y.J, L.Y.Zhao, Applying the evaluation indicator of total health expenditure to the policy analysis of the regional health expenditure, Chinese Journal of Health Policy, 2013,(11)6:49-55

[9] Y.Q.Li, Y.Jiang, Study on the health account in macro-economic in China, Chinese Health Economics, 2003,(11)5:16-18

[10] J.L.Lu, X.C.Xu, Q.Z, Review of methodology of health financing equity, Chinese Health Resources, 2012(4):4-10 